\title{
Weak Insertion of a Continuous Function between Two Comparable $\alpha$-Continuous (C-Continuous) Functions*
}

\section{Majid Mirmiran}

Department of Mathematics, University of Isfahan, Isfahan, Iran

Email: mirmir@sci.ui.ac.ir

Received 18 February 2016; accepted 4 March 2016; published 9 March 2016

Copyright (C) 2016 by author and OALib.

This work is licensed under the Creative Commons Attribution International License (CC BY). http://creativecommons.org/licenses/by/4.0/

(c) †) Open Access

\section{Abstract}

\section{A sufficient condition in terms of lower cut sets is given for the insertion of a continuous function between two comparable real-valued functions.}

\section{Keywords}

Weak Insertion, Strong Binary Relation, $C$-Open Set, Semi-Preopen Set, $\alpha$-Open Set, Lower Cut Set Subject Areas: Topology

\section{Introduction}

The concept of a $C$-open set in a topological space was introduced by E. Hatir, T. Noiri and S. Yksel in 1996 [1]. The authors define a set $S$ to be a $C$-open set if $S=U \cap A$, where $U$ is open and $A$ is semi-preclosed. A set $S$ is a $C$-closed set if its complement is $C$-open set or equivalently if $S=U \cup A$, where $U$ is closed and $A$ is semi-preopen. The authors show that a subset of a topological space is open if and only if it is an $\alpha$-open set and a $C$-open set. This enables them to provide the following decomposition of continuity: a function is continuous if and only if it is $\alpha$-continuous and $C$-continuous.

Recall that a subset $A$ of a topological space $(X, \tau)$ is called $\alpha$-open if $A$ is the difference of an open and a nowhere dense subset of $X$. A set $A$ is called $\alpha$-closed if its complement is $\alpha$-open or equivalently if $A$ is union of a closed and a nowhere dense set. Sets which are dense in some regular closed subspace are called semi-preopen or $\beta$-open. A set is semi-preclosed or $\beta$-closed if its complement is semi-preopen or $\beta$-open.

The concept of a set $A$ was $\beta$-open if and only if $A \subseteq C l(\operatorname{Int}(C l(A)))$ was introduced by J. Dontchev in 1998 [2].

\footnotetext{
${ }^{*}$ This work was supported by University of Isfahan and Centre of Excellence for Mathematics (University of Isfahan).
} 
Recall that a real-valued function $f$ defined on a topological space $X$ was called $A$-continuous if the preimage of every open subset of $\mathbb{R}$ belongs to $A$, where $A$ was a collection of subset of $X$ and this the concept was introduced by M. Przemski in 1993 [3]. Most of the definitions of function used throughout this paper are consequences of the definition of $A$-continuity. However, for unknown concepts, the reader might refer to papers introduced by J. Dontchev in 1995 [4], M. Ganster and I. Reilly in 1990 [5].

Hence, a real-valued function $f$ defined on a topological space $X$ is called $C$-continuous (resp. $\alpha$-continuous) if the preimage of every open subset of $\mathbb{R}$ is $C$-open (resp. $\alpha$-open) subset of $X$.

Results of Katětov in 1951 [6] and in 1953 [7] concerning binary relations and the concept of an indefinite lower cut set for a real-valued function, which was due to Brooks in 1971 [8], were used in order to give necessary and sufficient conditions for the strong insertion of a continuous function between two comparable real-valued functions.

If $g$ and $f$ are real-valued functions defined on a space $X$, we write $g \leq f$ in case $g(x) \leq f(x)$ for all $x$ in $X$.

The following definitions were modifications of conditions considered in paper introduced by E. Lane in 1976 [9].

A property $P$ defined relative to a real-valued function on a topological space is a c-property provided that any constant function has property $P$ and provided that the sum of a function with property $P$ and any continuous function also has property $P$. If $P_{1}$ and $P_{2}$ are $c$-property, the following terminology is used: A space $X$ has the weak c-insertion property for $\left(P_{1}, P_{2}\right)$ if and only if for any functions $g$ and $f$ on $X$ such that $g \leq f, g$ has property $P_{1}$ and $f$ has property $P_{2}$, then there exists a continuous function $h$ such that $g \leq h \leq f$.

In this paper, it is given a sufficient condition for the weak $c$-insertion property. Also several insertion theorems are obtained as corollaries of this result.

\section{The Main Result}

Before giving a sufficient condition for insertability of a continuous function, the necessary definitions and terminology are stated.

Let $(X, \tau)$ be a topological space, the family of all $\alpha$-open, $\alpha$-closed, $C$-open and $C$-closed will be denoted by $\alpha O(X, \tau), \alpha C(X, \tau), C O(X, \tau)$ and $C C(X, \tau)$, respectively.

Definition 2.1. Let $A$ be a subset of a topological space $(X, \tau)$. Respectively, we define the $\alpha$-closure, $\alpha$-interior, $C$-closure and $C$-interior of a set $A$, denoted by $\alpha \operatorname{Cl}(A), \alpha \operatorname{Int}(A), \operatorname{CCl}(A)$ and $\operatorname{CInt}(A)$ as follows:

$$
\begin{aligned}
& \alpha C l(A)=\cap\{F: F \supseteq A, F \in \alpha C(X, \tau)\} \\
& \alpha \operatorname{Int}(A)=\cup\{O: O \subseteq A, O \in \alpha O(X, \tau)\} \\
& C C l(A)=\cap\{F: F \supseteq A, F \in C C(X, \tau)\}
\end{aligned}
$$

and

$$
\operatorname{CInt}(A)=\cup\{O: O \subseteq A, O \in C O(X, \tau)\} .
$$

Respectively, we have $\alpha \operatorname{Cl}(A), \operatorname{CCl}(A)$ are $\alpha$-closed, semi-preclosed and $\alpha \operatorname{Int}(A), \operatorname{CInt}(A)$ are $\alpha$-open, semi-preopen.

The following first two definitions are modifications of conditions considered in [6] [7].

Definition 2.2. If $\rho$ is a binary relation in a set $S$ then $\bar{\rho}$ is defined as follows: $x \bar{\rho} y$ if and only if $y \rho v$ implies $x \rho v$ and $u \rho x$ implies $u \rho y$ for any $u$ and $v$ in $S$.

Definition 2.3. A binary relation $\rho$ in the power set $P(X)$ of a topological space $X$ is called a strong binary relation in $P(X)$ in case $\rho$ satisfies each of the following conditions:

1) If $A_{i} \rho B_{j}$ for any $i \in\{1, \cdots, m\}$ and for any $j \in\{1, \cdots, n\}$, then there exists a set $C$ in $P(X)$ such that $A_{i} \rho C$ and $C \rho B_{j}$ for any $i \in\{1, \cdots, m\}$ and any $j \in\{1, \cdots, n\}$.

2) If $A \subseteq B$, then $A \bar{\rho} B$.

3) If $A \rho B$, then $C l(A) \subseteq B$ and $A \subseteq \operatorname{Int}(B)$.

The concept of a lower indefinite cut set for a real-valued function was defined [8] as follows:

Definition 2.4. If $f$ is a real-valued function defined on a space $X$ and if 
$\{x \in X: f(x)<\ell\} \subseteq A(f, \ell) \subseteq\{x \in X: f(x) \leq \ell\}$ for a real number $\ell$, then $A(f, \ell)$ is called a lower indefinite cut set in the domain of $f$ at the level $\ell$.

We now give the following main result:

Theorem 2.1. Let $g$ and $f$ be real-valued functions on a topological space $X$ with $g \leq f$. If there exists a strong binary relation $\rho$ on the power set of $X$ and if there exist lower indefinite cut sets $A(f, t)$ and $A(g, t)$ in the domain of $f$ and $g$ at the level $t$ for each rational number $t$ such that if $t_{1}<t_{2}$ then $A\left(f, t_{1}\right) \rho A\left(g, t_{2}\right)$, then there exists a continuous function $h$ defined on $X$ such that $g \leq h \leq f$.

Proof. Let $g$ and $f$ be real-valued functions defined on $X$ such that $g \leq f$. By hypothesis there exists a strong binary relation $\rho$ on the power set of $X$ and there exist lower indefinite cut sets $A(f, t)$ and $A(g, t)$ in the domain of $f$ and $g$ at the level $t$ for each rational number $t$ such that if $t_{1}<t_{2}$ then $A\left(f, t_{1}\right) \rho A\left(g, t_{2}\right)$.

Define functions $F$ and $G$ mapping the rational numbers $\mathbb{Q}$ into the power set of $X$ by $F(t)=A(f, t)$ and $G(t)=A(g, t)$. If $t_{1}$ and $t_{2}$ are any elements of $\mathbb{Q}$ with $t_{1}<t_{2}$, then $F\left(t_{1}\right) \bar{\rho} F\left(t_{2}\right), G\left(t_{1}\right) \bar{\rho} G\left(t_{2}\right)$, and $F\left(t_{1}\right) \rho G\left(t_{2}\right)$. By Lemmas 1 and 2 of [7] it follows that there exists a function $H$ mapping $\mathbb{Q}$ into the power set of $X$ such that if $t_{1}$ and $t_{2}$ are any rational numbers with $t_{1}<t_{2}$, then $F\left(t_{1}\right) \rho H\left(t_{2}\right), H\left(t_{1}\right) \rho H\left(t_{2}\right)$ and $H\left(t_{1}\right) \rho G\left(t_{2}\right)$.

For any $x$ in $X$, let $h(x)=\inf \{t \in \mathbb{Q}: x \in H(t)\}$.

We first verify that $g \leq h \leq f$ : If $x$ is in $H(t)$ then $x$ is in $G\left(t^{\prime}\right)$ for any $t^{\prime}>t$; since $x$ is in $G\left(t^{\prime}\right)=A\left(g, t^{\prime}\right)$ implies that $g(x) \leq t^{\prime}$, it follows that $g(x) \leq t$. Hence $g \leq h$. If $x$ is not in $H(t)$, then $x$ is not in $F\left(t^{\prime}\right)$ for any $t^{\prime}<t$; since $x$ is not in $F\left(t^{\prime}\right)=A\left(f, t^{\prime}\right)$ implies that $f(x)>t^{\prime}$, it follows that $f(x) \geq t$. Hence $h \leq f$.

Also, for any rational numbers $t_{1}$ and $t_{2}$ with $t_{1}<t_{2}$, we have $h^{-1}\left(t_{1}, t_{2}\right)=\operatorname{Int}\left(H\left(t_{2}\right)\right) \backslash C l\left(H\left(t_{1}\right)\right)$. Hence $h^{-1}\left(t_{1}, t_{2}\right)$ is an open subset of $X$, i.e., $h$ is a continuous function on $X$.

The above proof used the technique of proof of Theorem 1 of [6].

\section{Applications}

The abbreviations $\alpha c$ and $C c$ are used for $\alpha$-continuous and $C$-continuous, respectively.

Corollary 3.1. If for each pair of disjoint $\alpha$-closed (resp. $C$-closed) sets $F_{1}, F_{2}$ of $X$, there exist open sets $G_{1}$ and $G_{2}$ of $X$ such that $F_{1} \subseteq G_{1}, F_{2} \subseteq G_{2}$ and $G_{1} \cap G_{2}=\varnothing$ then $X$ has the weak $c$-insertion property for $(\alpha c, \alpha c)$ (resp. $(C c, C c)$ ).

Proof. Let $g$ and $f$ be real-valued functions defined on the $X$, such that $f$ and $g$ are $\alpha c$ (resp. $C c$ ), and $g \leq f$. If a binary relation $\rho$ is defined by $A \rho B$ in case $\alpha \operatorname{Cl}(A) \subseteq \alpha \operatorname{Int}(B)$ (resp. $\operatorname{CCl}(A) \subseteq \operatorname{CInt}(B)$ ), then by hypothesis $\rho$ is a strong binary relation in the power set of $X$. If $t_{1}$ and $t_{2}$ are any elements of $\mathbb{Q}$ with $t_{1}<t_{2}$, then

$$
A\left(f, t_{1}\right) \subseteq\left\{x \in X: f(x) \leq t_{1}\right\} \subseteq\left\{x \in X: g(x)<t_{2}\right\} \subseteq A\left(g, t_{2}\right) ;
$$

since $\left\{x \in X: f(x) \leq t_{1}\right\}$ is an $\alpha$-closed (resp. C-closed) set and since $\left\{x \in X: g(x)<t_{2}\right\}$ is an $\alpha$-open (resp. $C$-open) set, it follows that $\alpha \operatorname{Cl}\left(A\left(f, t_{1}\right)\right) \subseteq \alpha \operatorname{Int}\left(A\left(g, t_{2}\right)\right)$ (resp. $\left.\operatorname{CCl}\left(A\left(f, t_{1}\right)\right) \subseteq \operatorname{CInt}\left(A\left(g, t_{2}\right)\right)\right)$. Hence $t_{1}<t_{2}$ implies that $A\left(f, t_{1}\right) \rho A\left(g, t_{2}\right)$. The proof follows from Theorem 2.1.

Corollary 3.2. If for each pair of disjoint $\alpha$-closed (resp. $C$-closed) sets $F_{1}, F_{2}$, there exist open sets $G_{1}$ and $G_{2}$ such that $F_{1} \subseteq G_{1}, \quad F_{2} \subseteq G_{2}$ and $G_{1} \cap G_{2}=\varnothing$ then every $\alpha$-continuous (resp. $C$-continuous) function is continuous.

Proof. Let $f$ be a real-valued $\alpha$-continuous (resp. $C$-continuous) function defined on the $X$. Set $g=f$, then by Corollary 3.1, there exists a continuous function $h$ such that $g=h=f$.

Corollary 3.3. If for each pair of disjoint subsets $F_{1}, F_{2}$ of $X$, such that $F_{1}$ is $\alpha$-closed and $F_{2}$ is $C$-closed, there exist open subsets $G_{1}$ and $G_{2}$ of $X$ such that $F_{1} \subseteq G_{1}, F_{2} \subseteq G_{2}$ and $G_{1} \cap G_{2}=\varnothing$ then $X$ have the weak $c$-insertion property for $(\alpha c, C c)$ and $(C c, \alpha c)$.

Proof. Let $g$ and $f$ be real-valued functions defined on the $X$, such that $g$ is $a c$ (resp. $C c$ ) and $f$ is $C c$ (resp. $a c$ ), with $g \leq f$. If a binary relation $\rho$ is defined by $A \rho B$ in case $\operatorname{CCl}(A) \subseteq \alpha \operatorname{Int}(B)$ (resp. $\alpha C l(A) \subseteq C \operatorname{Int}(B)$ ), then by hypothesis $\rho$ is a strong binary relation in the power set of $X$. If $t_{1}$ and $t_{2}$ are any elements of $\mathbb{Q}$ with $t_{1}<t_{2}$, then

$$
A\left(f, t_{1}\right) \subseteq\left\{x \in X: f(x) \leq t_{1}\right\} \subseteq\left\{x \in X: g(x)<t_{2}\right\} \subseteq A\left(g, t_{2}\right) ;
$$

since $\left\{x \in X: f(x) \leq t_{1}\right\}$ is a $C$-closed (resp. $\alpha$-closed) set and since $\left\{x \in X: g(x)<t_{2}\right\}$ is an $\alpha$-open (resp. 
$C$-open) set, it follows that $\operatorname{CCl}\left(A\left(f, t_{1}\right)\right) \subseteq \alpha \operatorname{Int}\left(A\left(g, t_{2}\right)\right)$ (resp. $\left.\alpha \operatorname{Cl}\left(A\left(f, t_{1}\right)\right) \subseteq \operatorname{CInt}\left(A\left(g, t_{2}\right)\right)\right)$. Hence $t_{1}<t_{2}$ implies that $A\left(f, t_{1}\right) \rho A\left(g, t_{2}\right)$. The proof follows from Theorem 2.1.

\section{Acknowledgements}

This research was partially supported by Centre of Excellence for Mathematics(University of Isfahan).

\section{References}

[1] Hatir, E., Noiri, T. and Yksel, S. (1996) A Decomposition of Continuity. Acta Mathematica Hungarica, 70, $145-150$. http://dx.doi.org/10.1007/BF00113919

[2] Dontchev, J. (1998) Between $\alpha$ - and $\beta$-Sets. Mathematica Balkanica, 12, 295-302.

[3] Przemski, M. (1993) A Decomposition of Continuity and $\alpha$-Continuity. Acta Mathematica Hungarica, 61, 93-98. http://dx.doi.org/10.1007/BF01872101

[4] Dontchev, J. (1995) The Characterization of Some Peculiar Topological Space via $\alpha$ - and $\beta$-Sets. Acta Mathematica Hungarica, 69, 67-71. http://dx.doi.org/10.1007/BF01874608

[5] Ganster, M. and Reilly, I. (1990) A Decomposition of Continuity. Acta Mathematica Hungarica, 56, $299-301$. http://dx.doi.org/10.1007/BF01903846

[6] Katětov, M. (1951) On Real-Valued Functions in Topological Spaces. Fundamenta Mathematicae, 38, 85-91.

[7] Katětov, M. (1953) Correction to, “On Real-Valued Functions in Topological Spaces”. Fundamenta Mathematicae, 40, 203-205.

[8] Brooks, F. (1971) Indefinite Cut Sets for Real Functions. The American Mathematical Monthly, 78, 1007-1010. http://dx.doi.org/10.2307/2317815

[9] Lane, E. (1976) Insertion of a Continuous Function. Pacific Journal of Mathematics, 66, 181-190. http://dx.doi.org/10.2140/pjm.1976.66.181 